\title{
PAPER \\ Performance Analysis of Repetition Coded OFDM Systems with Diversity Combining and Higher-Level Modulation*
}

\author{
Fumihito SASAMORI $^{\dagger \mathrm{a})}$, Member, Ziyan JIA ${ }^{\dagger}$, Student Member, Shiro HANDA ${ }^{\dagger}$, \\ and Shinjiro OSHITA ${ }^{\dagger}$, Members
}

\begin{abstract}
SUMMARY Orthogonal frequency division multiplexing (OFDM) communication systems have great advantages, such as high spectrum efficiency and robustness against multipath fading. In order to enhance the advantages, this paper investigates an efficient utilization of both diversity combining and higher-level modulation (adaptive modulation) with a repetition code on the frequency domain in the OFDM systems. The repetition coded OFDM systems can achieve an improvement of performance with such a simple structure as one pair of transmit/receive antennas. In this paper, we derive simple closed-form equations for bit error probability (BEP) and throughput, and then improvements of those performances in the proposed OFDM systems are verified by both theoretical analysis and Monte Carlo simulation.

key words: repetition coded OFDM, maximum ratio combining, higherlevel modulation, multipath fading, closed-form equation
\end{abstract}

\section{Introduction}

Orthogonal frequency division multiplexing (OFDM) has come to prominence in recent years because of high spectrum efficiency and robustness against multipath fading [1], [2]. Owing to the advantages, OFDM is applied to wireless LAN, terrestrial mobile communication, digital terrestrial TV broadcasting and so on. Meanwhile, OFDM is very fragile against Doppler spread and carrier frequency offset, which cause inter-subcarrier interference (ICI) and then degrade bit error probability (BEP). Many methods have been proposed to estimate the frequency fluctuations [3]-[5].

Adaptive modulation, in which a transmitter can adapt constellation size depending on the channel condition, and diversity combining, in which a transmitter and/or a receiver can adapt branch size, are both transmission techniques to combat fading. These two techniques are applicable to each digital-modulated symbol in the OFDM transmission [6], [7]. The former is attractive in improving throughput performance, whereas the latter aims to combat severe fading more effectively than the former.

As for the diversity combining, a simple kind of coded OFDM with a repetition code on the frequency domain, which is based on IEEE 802.16 protocols [8], [9], has been proposed. The repetition coded OFDM scheme brings many

\footnotetext{
Manuscript received November 30, 2009.

Manuscript revised July 8, 2010.

${ }^{\dagger}$ The authors are with the Faculty of Engineering, Shinshu University, Nagano-shi, 380-8553 Japan.

*This work was supported by MEXT, Grants-in-Aid for Young Scientists (B) 18760269.

a)E-mail: fsasa@shinshu-u.ac.jp

DOI: 10.1587/transcom.E94.B.194
}

good results, such as ICI cancellation [10], [11], blind channel estimation [12], and BEP improvement owing to diversity gain [11]. In [11], we have theoretically derived a simple closed-form equation for the BEP of differential quadrature phase-shift keying (DQPSK) in the repetition coded OFDM systems for the purpose of robustness against severe fading. In the proposed OFDM scheme, all subcarriers of OFDM are divided into two regions (low and high subcarrier frequencies), and DQPSK symbols are duplicated with half the subcarriers spacing. Owing to both double bit energy and frequency diversity gain, it can be theoretically verified that the BEP in the proposed systems is much improved as compared with that in the conventional DBPSK/OFDM systems on the conditions of the same carrier power and the same bit rate. But, from the standpoint of practical application, it is very important to consider coherent modulation like quadrature amplitude modulation (QAM) for the purpose of improving throughput performance.

In this paper, we further investigate an efficient utilization of higher-level modulation as well as diversity combining in the repetition coded OFDM systems. The information bit rate is reduced by half owing to the duplication, then the higher-level modulation should be considered in the proposed OFDM systems to keep the bit rate. In order to theoretically verify the advantage of the proposed scheme, we derive simple closed-form equations for the BEP and the throughput of PSK and QAM. Many performance analyses of the OFDM systems over fading channels have been proposed, for example [13], [14]. But few of them analyze the effects of both the diversity combining and the higher-level modulation, what is more, with such a simple structure as one pair of transmit/receive antennas. A simple transmit diversity technique [15] and its application to OFDM, spacetime coded OFDM [16] and space-frequency coded OFDM [17], have been proposed. Those techniques can easily provide the same diversity gain as maximum ratio combining (MRC), but require multiple transmit antennas. On the other hand, the proposed OFDM can provide the limited diversity gain which depends on the frequency selectivity, but requires only one transmit antenna. Finally, the improvements of BEP and throughput performances are verified by both theoretical analysis and Monte Carlo simulation.

The remainder of this paper is organized as follows. In Sect. 2, a system model of the proposed OFDM systems is described. In Sect. 3, simple closed-form equations for the $\mathrm{BEP}$ and the throughput are derived. We evaluate the perfor- 
mances of both conventional and proposed OFDM systems in Sect. 4. The paper is concluded in Sect. 5.

\section{System Model}

Figure 1 shows a block diagram of the OFDM systems with a repetition code on the frequency domain, where $N_{c}$ is the number of subcarriers. At a transmitter, a binary data sequence is converted to digital-modulated symbols $S_{i}$ $\left(i=0,1, \cdots, N_{c} / 2-1\right)$, which are parallelized by serialto-parallel ( $\mathrm{S} / \mathrm{P})$ conversion and duplicated with $N_{c} / 2$ subcarriers spacing. The information bit rate is reduced by half owing to the duplication, then the higher-level modulation is considered in the proposed OFDM systems to keep the bit rate. After OFDM modulation (IFFT), parallel-to-serial $(\mathrm{P} / \mathrm{S})$ conversion, guard interval $(\mathrm{GI})$ insertion and digitalto-analog (D/A) conversion, generated OFDM symbols are transmitted.

OFDM symbols are subjected to Rayleigh fading and are added white Gaussian noise (AWGN). The fading channel is both time- and frequency-selective, but it is assumed that the channel remains constant over one OFDM symbol duration [5] and inter-symbol interference (ISI) caused by delay spread can be completely avoided with the GI, which is longer than the maximum multipath delay. In Monte Carlo simulation mentioned later, a tapped-delay line channel simulator is considered as the fading channel [18], and each channel tap is simulated using the Jakes' model [19].

A receiver is constructed by reversing the process of the transmitter as follows: analog-to-digital (A/D) conversion, GI deletion, S/P conversion, OFDM demodulation (FFT), coherent detection, maximum ratio combining (MRC) for two symbols with $N_{c} / 2$ subcarriers spacing, $\mathrm{P} / \mathrm{S}$ conversion and data decision. At the diversity combiner, full diversity gain cannot be achieved because of frequency correlation between subcarriers, but we will verify the advantage of the proposed scheme even as for the imperfect diversity gain. It should be noted that the frequency diversity combining can be easily replaced by the time diversity combining with a time interval.

In this paper, the proposed scheme will be compared with the conventional one without duplication and MRC on the conditions of the same carrier power and the same bit rate. Table 1 groups Gray-mapped $M$-PSK and $M$-QAM considering the same bit rate per symbol $R$, which is defined as

$$
R= \begin{cases}\log _{2} M & \text { (for conventional OFDM), } \\ \frac{1}{2} \log _{2} M & \text { (for proposed OFDM). }\end{cases}
$$

In the next section, we will theoretically analyze the effects of both the correlated diversity combining and the higherlevel modulation upon BEP and throughput performances. That is, it will be theoretically verified that the impact of the correlated diversity gain is larger than the decrease of power efficiency by the higher-level modulation. Generally speaking, more accurate detection is needed when the constellation size of modulation is increased. But the purpose of this study is to analyze the optimal effects of both the diversity combining and the higher-level modulation, then the ideal coherent detection is assumed in this paper.

\section{Performance Analysis}

One of the important matters to analyze the performances is the correlated frequency diversity effect, which has been derived in [11]. The derived frequency correlation function is very simple, but includes a computational error when a frequency spacing is large [20]. Therefore, after introducing a modified and accurate frequency correlation function, simple closed-form equations for the BEP and the throughput will be derived.

\subsection{Modified Frequency Correlation Function}

We have derived the absolute frequency correlation function as follows: [11]

$$
\left|\rho_{B}(l)\right|=\frac{1}{\sqrt{1+\left\{2 \pi l(1+\alpha) \frac{\sigma_{\tau}}{T_{s}}\right\}^{2}}},
$$

where $\sigma_{\tau} / T_{s}$ is the rms delay spread normalized by the OFDM symbol duration, $\alpha$ is the ratio of GI duration $T_{g}$ to data symbol duration $T_{d}\left(\alpha=T_{g} / T_{d}\right)$, and $l$ is the number of subcarriers spacing, for example, $l=N_{c} / 2$ in Fig. 1 .

Table 1 Subcarrier modulations with same bit rate.

\begin{tabular}{c||c|c|c|c}
\hline \hline$R$ & 1 & 2 & 3 & 4 \\
\hline \hline $\begin{array}{c}\text { Conventional } \\
\text { OFDM }\end{array}$ & BPSK & QPSK & 8PSK & 16QAM \\
\hline $\begin{array}{c}\text { Proposed } \\
\text { OFDM }\end{array}$ & QPSK & 16QAM & 64QAM & 256QAM \\
\hline \hline
\end{tabular}

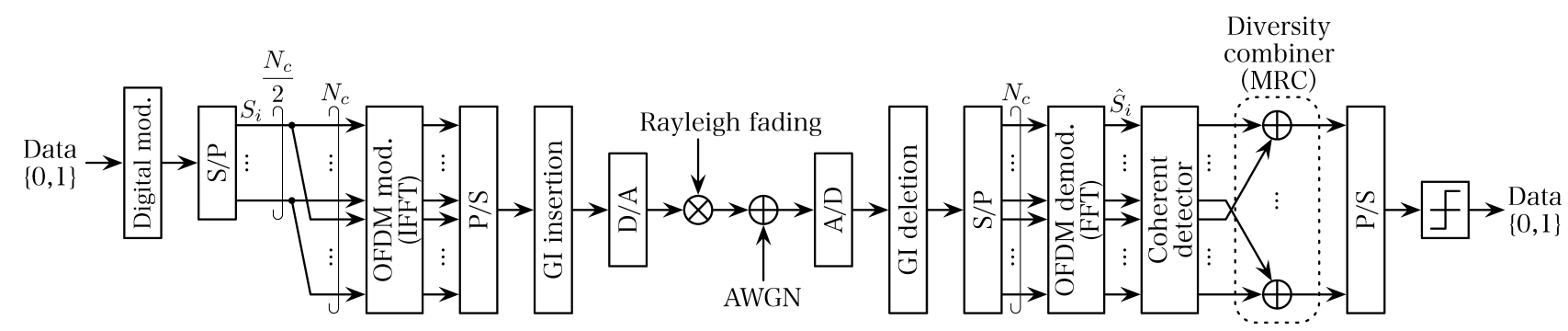

Fig. 1 System model in equivalent low-pass system. 


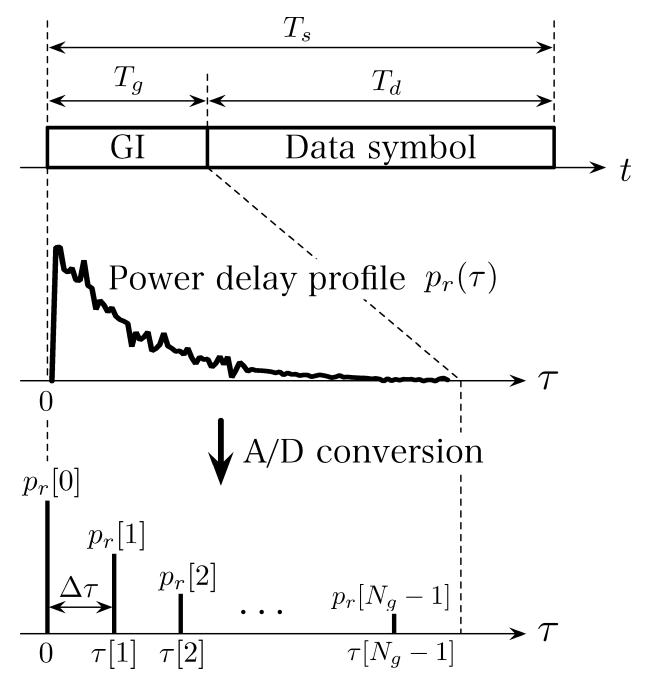

Fig. 2 Discrete-time power delay profile after A/D conversion.

Generally speaking, a frequency correlation function is derived by Fourier transform of a power delay profile, which is a continuous-time function. Equation (2) has also been derived by the above method, but owing to the $\mathrm{A} / \mathrm{D}$ conversion at the OFDM receiver, the frequency correlation between subcarriers should be derived from a discrete-time power delay profile by discrete Fourier transform. Moreover, at the price of simplicity, (2) holds only in the case of an exponential decay power delay profile. Therefore, for the strictness and generality, a modified and accurate frequency correlation function is introduced in this section.

Figure 2 shows the concept of discrete-time power delay profile. At the A/D converter with FFT sampling period $\Delta \tau$, the continuous-time profile $p_{r}(\tau)$ within GI is converted into the discrete-time profile with the power $p_{r}[n]$ in the time delay $\tau[n]\left(n=0,1, \cdots, N_{g}-1\right)$. Equation (2) is originally derived by the Fourier transform as follows: [18]

$$
\left|\rho_{B}(\Delta f)\right|=\frac{\left|\int_{0}^{\infty} p_{r}(\tau) \exp (-j 2 \pi \Delta f \tau) d \tau\right|}{P_{r}},
$$

where $\Delta f$ and $P_{r}$ are a frequency spacing and total power of $p_{r}(\tau)$, respectively. Since the frequency spacing between adjacent subcarriers is $1 / T_{d}, \Delta f$ with $l$ subcarriers spacing in (3) becomes

$$
\Delta f=\frac{l}{T_{d}}=\frac{l(1+\alpha)}{T_{s}} .
$$

When converting (3) into the discrete Fourier transform and limiting the maximum time delay to $T_{g}$, the modified frequency correlation function can be expressed as

$$
\left|\rho_{B}(l)\right|=\frac{\left|\sum_{n=0}^{N_{g}-1} p_{r}[n] \exp \left\{-j 2 \pi \frac{l(1+\alpha)}{T_{s}} \tau[n]\right\} \Delta \tau\right|}{\sum_{n=0}^{N_{g}-1} p_{r}[n] \Delta \tau}
$$

\begin{tabular}{|c|c|c|}
\hline FFT point size $N_{d}$ & \multicolumn{2}{|c|}{64,256} \\
\hline GI sample size $N_{g}$ & \multicolumn{2}{|c|}{16,64} \\
\hline Channel model & $\begin{array}{c}\text { Exponential } \\
\text { decay }\end{array}$ & $\begin{array}{c}\text { ETSI/BRAN } \\
\text { channel-A }\end{array}$ \\
\hline $\begin{array}{l}\text { rms delay spread } \\
\sigma_{\tau} / T_{s}\end{array}$ & $0.002,0.01$ & 0.007 \\
\hline$=\mid \sum_{n=0}^{N_{g}-1} p_{r}^{\prime}[n]$ & $\{-j 2 \pi l($ & $\left.\alpha) \tau^{\prime}[n]\right\} \mid$ \\
\hline
\end{tabular}

Table 2 Simulation parameters (1).

where $p_{r}^{\prime}[n]$ is each power normalized by total power and $\tau^{\prime}[n]$ is each delay time normalized by OFDM symbol duration as follows:

$$
\begin{aligned}
& p_{r}^{\prime}[n]=\frac{p_{r}[n]}{\sum_{n^{\prime}=0}^{N_{g}-1} p_{r}\left[n^{\prime}\right]}, \\
& \tau^{\prime}[n]=\frac{\tau[n]}{T_{s}} .
\end{aligned}
$$

Now we justify the modified function (5) with Monte Carlo simulation. In the simulation, we calculate the expected value of correlation between two symbols $\hat{S}_{0}$ in the zeroth subcarrier and $\hat{S}_{l}$ in the $l$-th subcarrier after OFDM demodulation (FFT), that is,

$$
\left|\rho_{B}(l)\right|_{\text {simu }}=\left|E\left[\frac{\hat{S}_{0}^{*} \hat{S}_{l}}{\hat{S}_{0}^{*} \hat{S}_{0}}\right]\right|,\left(l=0,1, \cdots, N_{d}-1\right),
$$

where $N_{d}$ is FFT point size. Table 2 shows the simulation parameters, where the exponential decay model and the ETSI/BRAN channel-A model [21], [22] are assumed as the power delay profile based on the tapped-delay line model [18]. Figure 3 shows frequency correlation characteristics as a function of the subcarrier spacing. It is found from the figure that the modified function (5) can precisely express the characteristics, whereas the simple function (2) includes a computational error when using the large frequency spacing in the proposed OFDM systems. By considering sampling effect, the frequency correlation characteristic becomes periodic and concave, because the output from FFT $\hat{S}_{l}$ is periodic with period $N_{d}$ and the first output is continuous with the last one. The characteristic becomes closer to the value from the simple function (2) in the case of large FFT point size $N_{d}$, because discrete-time Fourier transform can be approximated to continuous-time one by enlarging the sampling frequency.

\subsection{Average Bit Error Probability}

Now we derive a theoretical equation for the BEP over fading channels by using the absolute frequency correlation function $\left|\rho_{B}(l)\right|$. Fast of all, a theoretical equation for the BEP with Gray-mapped $M$-PSK or $M$-QAM over AWGN channels $P_{b}(\gamma)$ is given by [23] 


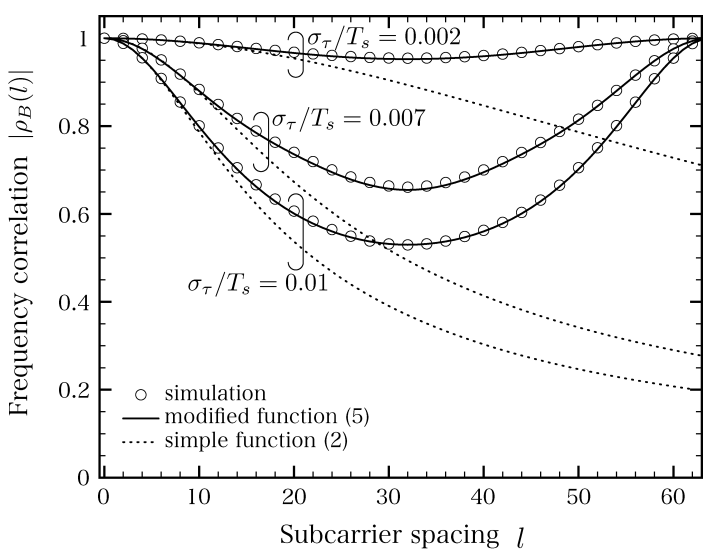

(a) $N_{d}=64, N_{g}=16$

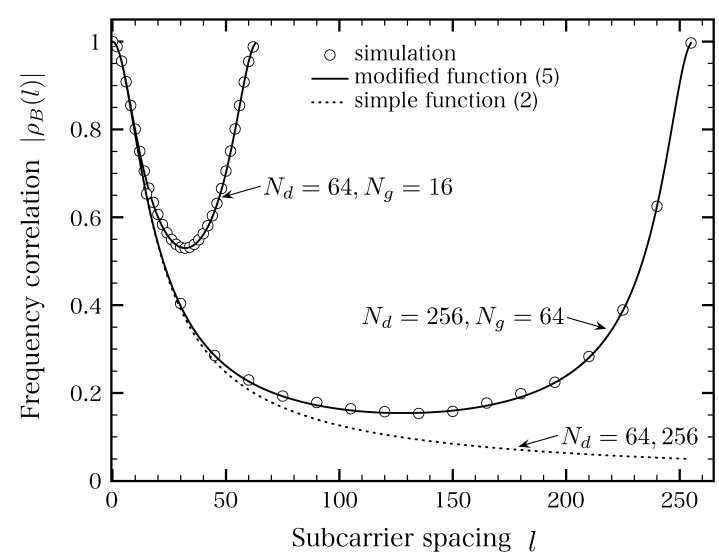

(b) $\sigma_{\tau} / T_{s}=0.01$

Fig. 3 Frequency correlation characteristics as a function of subcarrier spacing.

$$
P_{b}(\gamma)=A_{i} \operatorname{erfc}\left(\sqrt{B_{i} \gamma}\right), \quad\left(i=\log _{2} M\right),
$$

where $\gamma$ is carrier-to-noise ratio (CNR) of received signal and $\operatorname{erfc}(\cdot)$ is a complementary error function. The values of $A_{i}$ and $B_{i}$ in each modulation are appeared in Table 3. By averaging (8) with a probability density function (PDF) of CNR $p(\gamma)$, the average $\operatorname{BEP} \bar{P}_{b}(\Gamma)$ can be derived, i.e.,

$$
\bar{P}_{b}(\Gamma)=\int_{0}^{\infty} P_{b}(\gamma) p(\gamma) d \gamma,
$$

where $\Gamma$ is the average $\mathrm{CNR}$, i.e.,

$$
\Gamma=\int_{0}^{\infty} \gamma p(\gamma) d \gamma .
$$

\subsubsection{BEP Equation in Conventional OFDM Systems}

The PDF of a Rayleigh random variable can be expressed as [24]

$$
p(\gamma)=\frac{1}{\Gamma} \exp \left(-\frac{\gamma}{\Gamma}\right)
$$

Substituting (8) and (11) into (9), the average BEP over
Table 3 Values of $A_{i}$ and $B_{i}$

\begin{tabular}{c|c|c|c|c}
\hline \hline$M$ & $i$ & Modulation & $A_{i}$ & $B_{i}$ \\
\hline \hline 2 & 1 & BPSK & $1 / 2$ & 1 \\
\hline 4 & 2 & QPSK & $1 / 2$ & $1 / 2$ \\
\hline 8 & 3 & 8PSK & $1 / 3$ & $\sin ^{2}(\pi / 8)$ \\
\hline 16 & 4 & 16QAM & $3 / 8$ & $1 / 10$ \\
\hline 64 & 6 & 64QAM & $7 / 24$ & $1 / 42$ \\
\hline 256 & 8 & 256QAM & $15 / 64$ & $1 / 170$ \\
\hline \hline
\end{tabular}

Rayleigh fading channels becomes

$$
\bar{P}_{b}(\Gamma)=A_{i}\left(1-\frac{1}{\sqrt{1+\frac{1}{B_{i} \Gamma}}}\right) .
$$

It is noted that the following integration result is utilized to derive (12):

$$
\begin{gathered}
\int_{0}^{\infty} x \cdot \exp \left(-a x^{2}\right) \cdot \operatorname{erfc}(b x) d x \\
=\frac{1}{2 a}\left(1-\frac{b}{\sqrt{a+b^{2}}}\right) .
\end{gathered}
$$

The BEP in the conventional OFDM systems without duplication and MRC can be calculated with (12) and Table 3.

\subsubsection{BEP Equation in Proposed OFDM Systems}

The PDF of CNR when combining correlated two signals with MRC over Rayleigh fading channels can be expressed as [24]

$$
\begin{aligned}
p(\gamma)=\frac{1}{2\left|\rho_{B}(l)\right| \Gamma} & {\left[\exp \left\{-\frac{\gamma}{\left(1+\left|\rho_{B}(l)\right|\right) \Gamma}\right\}\right.} \\
& \left.-\exp \left\{-\frac{\gamma}{\left(1-\left|\rho_{B}(l)\right|\right) \Gamma}\right\}\right] .
\end{aligned}
$$

Substituting (8) and (14) into (9) and using (13), the average BEP becomes

$$
\begin{aligned}
\bar{P}_{b}(\Gamma)=A_{i}[ & 1-\frac{1}{2\left|\rho_{B}(l)\right|}\left\{\frac{1+\left|\rho_{B}(l)\right|}{\sqrt{1+\frac{1}{\left(1+\left|\rho_{B}(l)\right|\right) B_{i} \Gamma}}}\right. \\
& \left.\left.-\frac{1-\left|\rho_{B}(l)\right|}{\sqrt{1+\frac{1}{\left(1-\left|\rho_{B}(l)\right|\right) B_{i} \Gamma}}}\right\}\right] .
\end{aligned}
$$

The BEP in the proposed OFDM systems with correlated frequency diversity combining can be calculated with (15) and Table 3.

When the frequency spacing and/or the rms delay spread are very large, that is, when two signals with MRC can be considered to be mutually independent, the maximum diversity gain can be achieved. In this case, the PDF of CNR can be expressed as [24]

$$
p(\gamma)=\frac{\gamma}{\Gamma^{2}} \exp \left(-\frac{\gamma}{\Gamma}\right)
$$


Substituting (8) and (16) into (9), the average BEP becomes

$$
\bar{P}_{b}(\Gamma)=A_{i}\left\{1-\frac{1+\frac{3}{2 B_{i} \Gamma}}{\left(1+\frac{1}{B_{i} \Gamma}\right)^{\frac{3}{2}}}\right\} .
$$

It is noted that the following integration result is utilized to derive (17):

$$
\begin{gathered}
\int_{0}^{\infty} x^{3} \cdot \exp \left(-a x^{2}\right) \cdot \operatorname{erfc}(b x) d x \\
=\frac{1}{2 a^{2}}\left\{1-\frac{b\left(3 a+2 b^{2}\right)}{2\left(a+b^{2}\right)^{\frac{3}{2}}}\right\}
\end{gathered}
$$

Equation (17) can calculate the best BEP performance in the proposed OFDM systems.

Finally, the frequency diversity combining can be easily replaced by the time diversity combining with a time interval, that is, by replacing the frequency correlation function $\left|\rho_{B}(l)\right|$ with the time correlation function

$$
\left|\rho_{B}^{\prime}\left(l^{\prime}\right)\right|=\left|J_{0}\left(2 \pi l^{\prime} f_{D} T_{s}\right)\right|,
$$

where $f_{D}$ and $l^{\prime}$ are the maximum Doppler frequency and the time interval (the number of OFDM symbols), respectively, and $J_{0}(\cdot)$ is a Bessel function of the first kind of zeroth order.

\subsection{Average Throughput}

Since the proposed scheme aims to improve the throughput performance as well as the BEP performance, we theoretically analyze the average throughput by using the derived average BEP $\bar{P}_{b}(\Gamma)$. The average throughput in terms of bits per second per Hertz can be defined as

$$
\bar{S}(\Gamma)=R\left(\frac{1}{1+\alpha}\right) \bar{P}_{p}(\Gamma),
$$

where $1 /(1+\alpha)$ means the loss by GI insertion, $\bar{P}_{p}(\Gamma)$ is the average packet success probability with a packet size (the number of bits per packet) $D$ as follows:

$$
\bar{P}_{p}(\Gamma)=\left\{1-\bar{P}_{b}(\Gamma)\right\}^{D} .
$$

In the throughput analysis, an optimal bit interleaver is assumed to randomize burst bit errors.

\section{Performance Evaluation}

We theoretically verify the effectiveness of the OFDM systems employing the correlated diversity combining and the higher-level modulation described in Sect. 2. The proposed scheme should be compared with the conventional one without duplication and MRC on the conditions of the same carrier power and the same bit rate in Table 1 . In order to justify the proposed analysis in Sect. 3, the performance obtained from Monte Carlo simulation is also evaluated. Table 4 shows the simulation parameters.
Table 4 Simulation parameters (2).

\begin{tabular}{c|c}
\hline \hline Modulation & PSK, QAM \\
\hline Detection & Coherent (ideal) \\
\hline FFT point size $N_{d}$ & 64 \\
\hline Subcarrier size $N_{c}$ & 48 \\
\hline GI sample size $N_{g}$ & $16(\alpha=1 / 4)$ \\
\hline Noise & AWGN \\
\hline Fading & Rayleigh \\
\hline Channel model & Exponential decay \\
\hline \hline
\end{tabular}

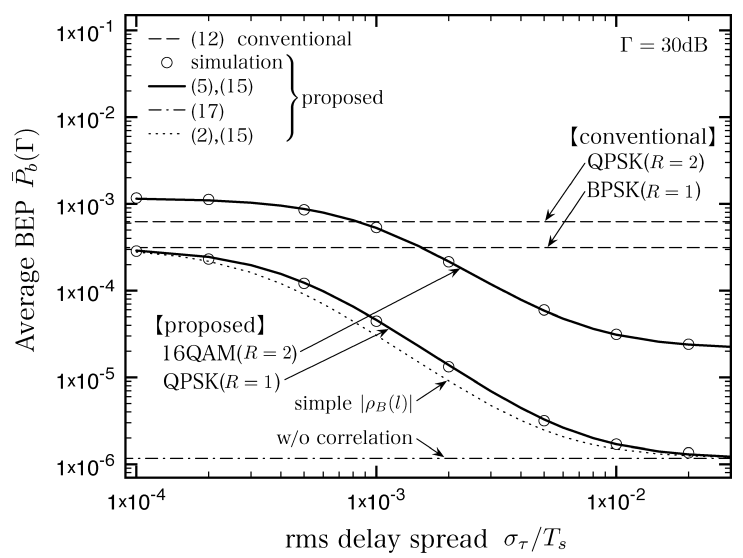

(a) $R=1, R=2$

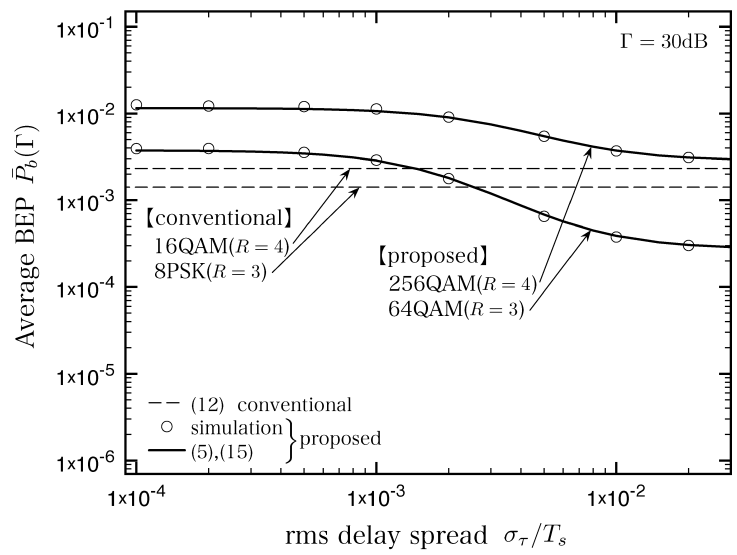

(b) $R=3, R=4$

Fig. 4 Average BEP versus rms delay spread when average CNR $\Gamma$ is $30 \mathrm{~dB}$

\subsection{Average Ber Performance}

Figure 4 shows the average BEP performances as a function of the rms delay spread when the average CNR $\Gamma$ is $30 \mathrm{~dB}$. The following analytical results of QPSK in the proposed OFDM systems are simultaneously indicated in Fig. 4(a): the BEP with the simple frequency correlation function (2) instead of (5) and the best BEP with uncorrelated MRC in (17). It is noted that the performance of the conventional scheme is irrelevant to the rms delay spread, then only analytical results are indicated in the figure. We first point out the accuracy of the modified frequency correlation function (5) in the performance. It can be observed from 


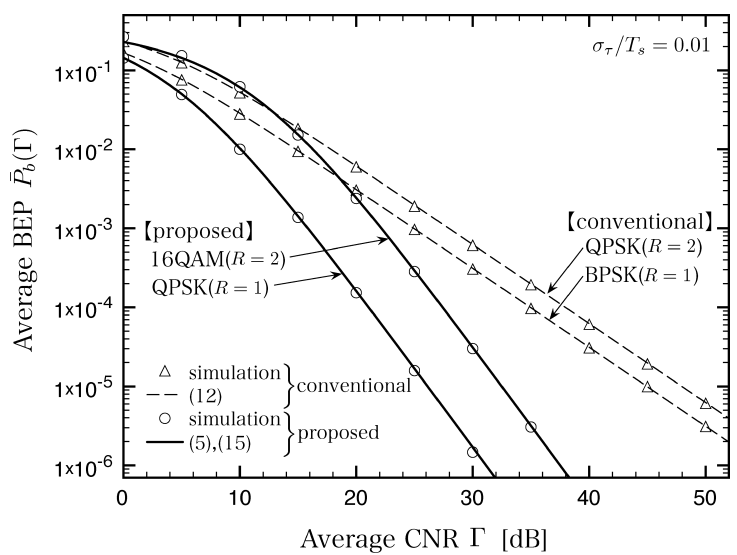

(a) $R=1, R=2$

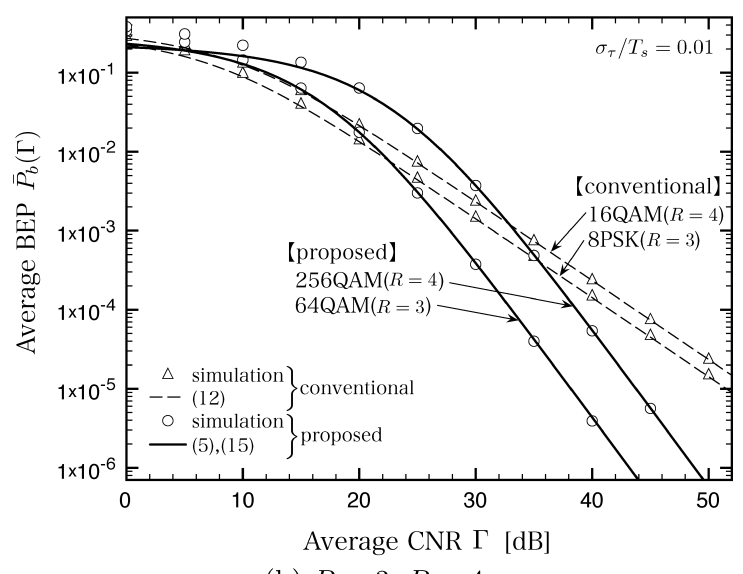

(b) $R=3, R=4$

Fig. 5 Average BEP versus average CNR when rms delay spread $\sigma_{\tau} / T_{s}$ is 0.01 .

the figure that the proposed scheme can provide the better performance with increasing the rms delay spread $\sigma_{\tau} / T_{s}$. That is because the frequency correlation between subcarriers becomes small, which enlarges the frequency diversity gain and improves the BEP. Owing to this fact, the proposed scheme outperforms the conventional one when $\sigma_{\tau} / T_{s}>8 \times 10^{-4}$ for $R=2$ and $\sigma_{\tau} / T_{s}>2 \times 10^{-3}$ for $R=3$. The proposed scheme always outperforms for $R=1$, whereas the reverse occurs for $R=4$. The situation changes according to the CNR except for $R=1$. The proposed scheme can utilize double bit energy to demodulate data as compared with the conventional one, whereas QPSK can utilize half bit energy as compared with BPSK in the same CNR condition. In the case of small delay spread (with no diversity gain), the proposed QPSK and the conventional BPSK consequently utilize the same bit energy to demodulate data and show almost the same performance at any CNR. When the delay spread becomes large, the proposed scheme can acquire the frequency diversity gain and then the performance is improved. The proposed QPSK consequently outperforms the conventional BPSK at any delay spread.

Figure 5 shows the average BEP performances as a

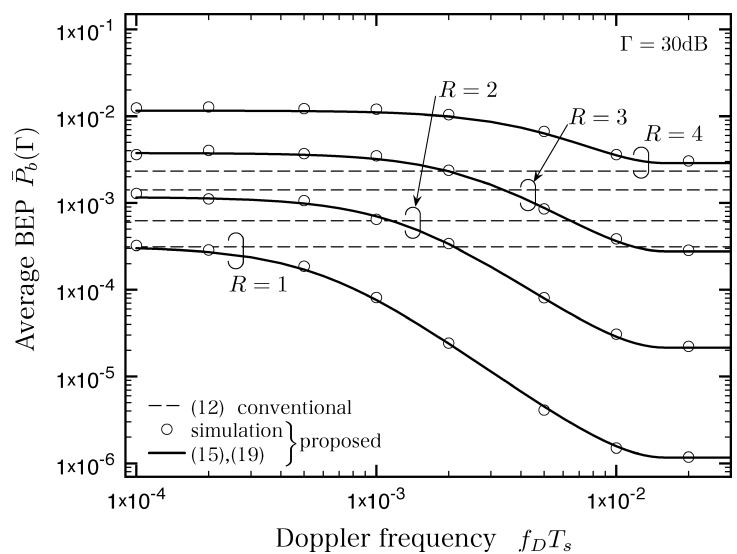

Fig. 6 Average BEP versus Doppler frequency with time diversity combining $\left(l^{\prime}=24\right)$ when average CNR $\Gamma$ is $30 \mathrm{~dB}$.

function of the average CNR when the rms delay spread $\sigma_{\tau} / T_{s}$ is 0.01 . It can be observed from the figure that the slope of BEP curve improves significantly owing to the frequency diversity gain. Meanwhile, the higher-level modulation has a double-edged property: decrease of power efficiency and increase of frequency efficiency. Therefore, the proposed scheme outperforms the conventional one under high CNR condition, concretely speaking, when $\Gamma>12 \mathrm{~dB}$ for $R=2, \Gamma>22 \mathrm{~dB}$ for $R=3$, and $\Gamma>33 \mathrm{~dB}$ for $R=4$. The frequency efficiency, that is, the throughput performance will be discussed later.

Figure 6 shows the average BEP performance as a function of the Doppler frequency with the time diversity combining. The analytical results are calculated with the time correlation function (19) instead of the frequency correlation function (5). The characteristics in Figs. 4 and 6 are very similar, because relative merits of both schemes depend on the value of fading correlation, irrelevant whether frequency or time correlation.

\subsection{Average Throughput Performance}

Figure 7 shows the average throughput performances as a function of the average CNR when the rms delay spread $\sigma_{\tau} / T_{s}$ is 0.01 and the packet size $D$ is 100 bits. The results indicate that the proposed scheme can provide the better performance at any bit rate $R$ in that the lower CNR is sufficient to achieve the maximum throughput. Meanwhile, especially when $R=4$, the conventional scheme outperforms under low CNR condition due to the power efficiency of the lower-level modulation. But the gap can be filled with increasing the packet size $D$.

Figure 8 theoretically compares the required $\mathrm{CNR} \Gamma_{\text {req }}$ for $90 \%$ maximum throughput as a function of the packet size when $\sigma_{\tau} / T_{s}$ is 0.01 . It can be observed from the figure that the increase of $\Gamma_{\text {req }}$ with enlarging the packet size $D$ in the proposed systems is modest. This is because the average throughput performance mainly depends on the average packet success probability $\bar{P}_{p}(\Gamma)$ in $(21)$ and the advantage of the BEP performance $\bar{P}_{b}(\Gamma)$ in the proposed sys- 


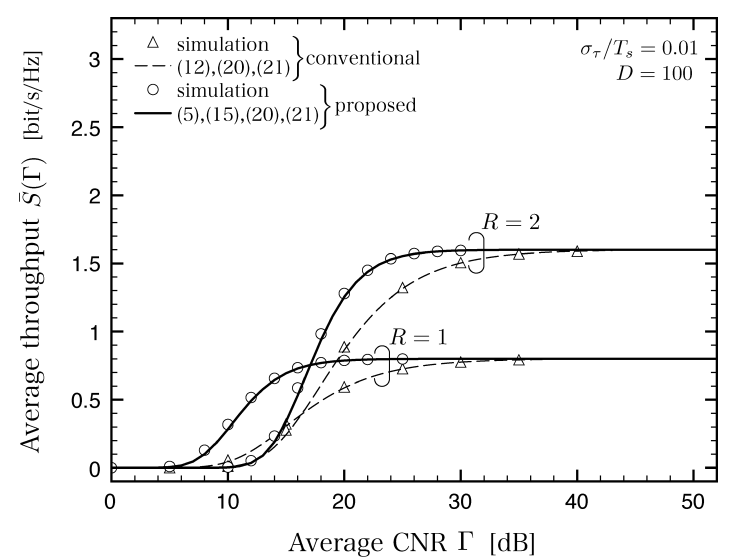

(a) $R=1, R=2$

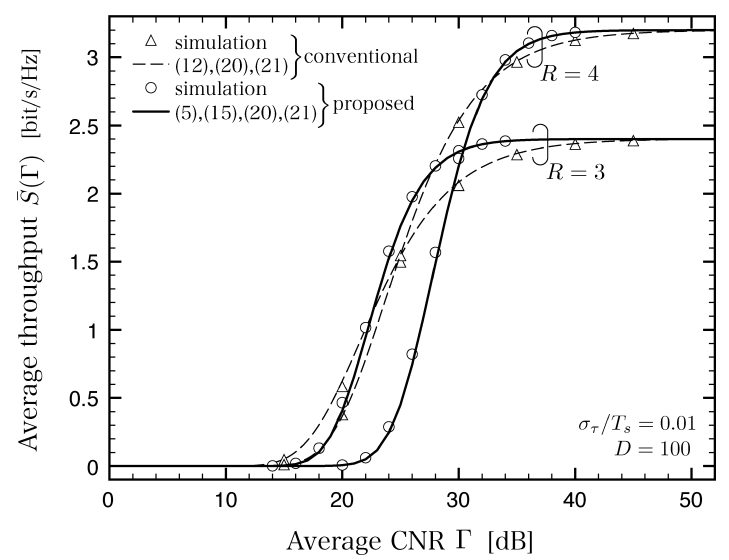

(b) $R=3, R=4$

Fig. 7 Average throughput versus average CNR when rms delay spread $\sigma_{\tau} / T_{s}$ is 0.01 and packet size $D$ is 100 bits.

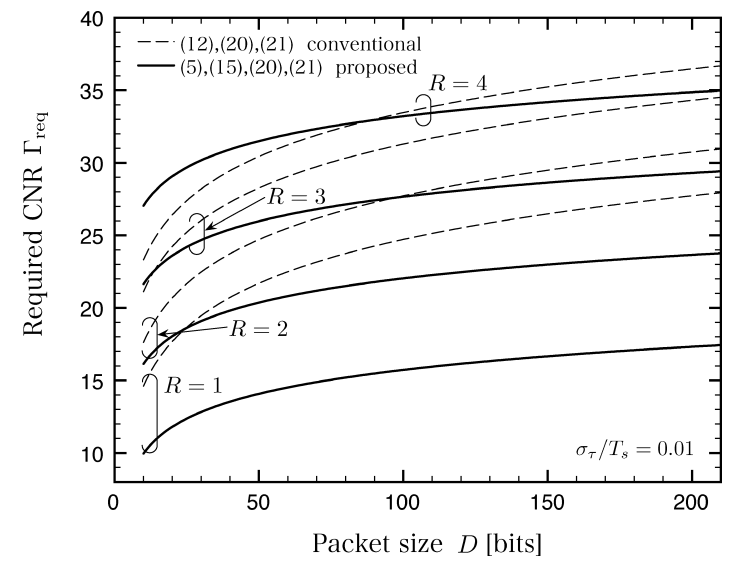

Fig. 8 Required CNR for $90 \%$ maximum throughput versus packet size when rms delay spread $\sigma_{\tau} / T_{s}$ is 0.01 .

tems appears more evidently when enlarging the packet size $D$. Figure 9 theoretically compares the $\Gamma_{\text {req }}$ as a function of the rms delay spread when $D$ is 100 bits. It can be observed from the figure that the proposed scheme outperforms at any $\sigma_{\tau} / T_{s}$ for $R=1$, whereas requires the larger delay spread (diversity gain) with increasing $R$.

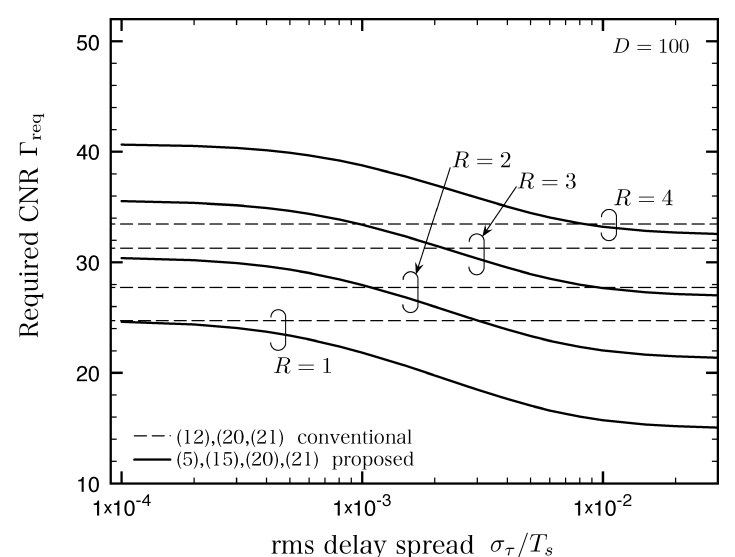

Fig. 9 Required CNR for $90 \%$ maximum throughput versus rms delay spread when packet size $D$ is 100 bits.

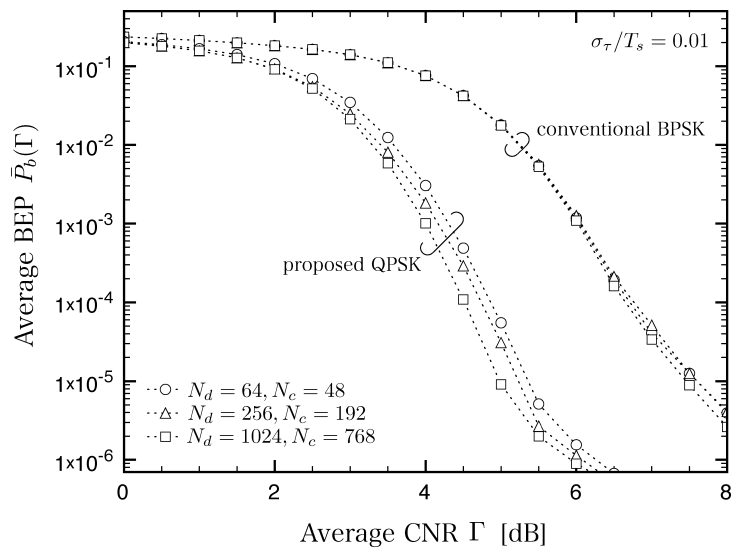

(a) Average BEP versus average CNR.

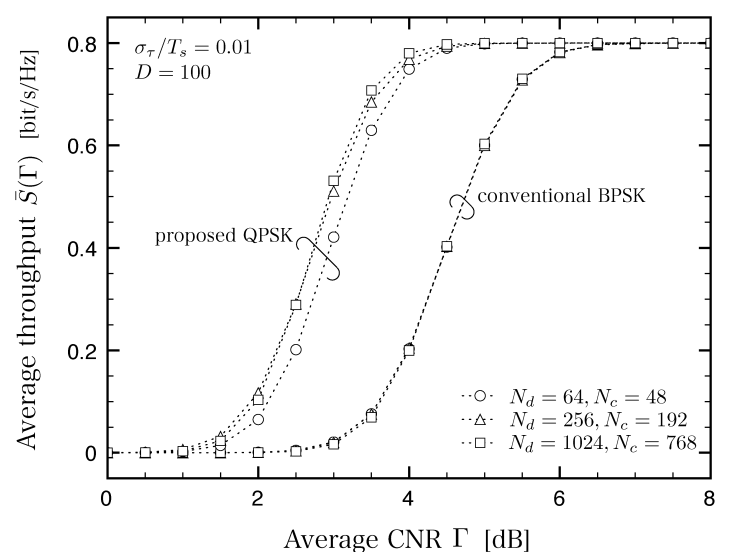

(b) Average throughput versus average CNR.

Fig. 10 Performances with turbo code in the case of $R=1$ when rms delay spread $\sigma_{\tau} / T_{s}$ is 0.01 and packet size $D$ is 100 bits.

Finally, we would like to emphasize in Figs. 4-7 that the simulation results confirm the theoretical performance analysis in Sect. 3. 


\subsection{Application of FEC to Proposed OFDM Systems}

As an application of the proposed OFDM systems, we briefly discuss the additional effect of forward error correction (FEC) with the simulation. As for FEC coded OFDM, Reed-Solomon (RS) coded OFDM [13], turbo coded OFDM [14], and low-density parity-check (LDPC) coded OFDM [25] have been proposed. It is already known by [17] that the OFDM with diversity technique (the space-frequency coded OFDM) can outperform the RS coded OFDM, then we briefly show the advantage of both turbo coded and repetition coded OFDM systems in the case of $R=1$. We use popular rate- $1 / 2$ punctured turbo code which consists of two recursive systematic convolutional encoders with constraint length of 3 , generator polynomial $(7,5)$, and interleaver size of 10000 concatenated in parallel. We also use the BCJR algorithm as a decoding method.

Figure 10 shows the average BEP and throughput performances with turbo code when the rms delay spread $\sigma_{\tau} / T_{s}$ is 0.01 and the packet size $D$ is 100 bits. It is found from the figure that the performances of both the conventional BPSK and the proposed QPSK are significantly improved owing to the turbo code, and that the proposed QPSK has the additional advantage of the frequency diversity gain. As shown in Fig. 3(b), the frequency correlation between symbols with half the subcarriers spacing becomes low with increasing the FFT point size $N_{d}$ in the same delay spread $\sigma_{\tau} / T_{s}$, which improves the performances of the proposed QPSK.

\section{Conclusion}

In this paper, we introduced a repetition coded OFDM employing diversity combining and higher-level modulation with such a simple structure as one pair of transmit/receive antennas. In order to compare the proposed scheme with the conventional one on the conditions of the same carrier power and the same bit rate, we derived simple closed-form equations for bit error probability and throughput. The effectiveness of the proposed OFDM systems was verified by both theoretical analysis and Monte Carlo simulation.

We did not theoretically analyze the effect of FEC codes in this paper, because the performances vary depending on the structures of encoder and decoder, interleaving size and so on, whose evaluations are beyond the scope of this paper. As future works, we would like to analyze FEC coded OFDM systems.

\section{References}

[1] S.B. Weinstein and P.M. Ebert, "Data transmission by frequencydivision multiplexing using the discrete Fourier transform," IEEE Trans. Commun., vol.COM-19, no.5, pp.628-634, Oct. 1971.

[2] J.A.C. Bingham, "Multicarrier modulation for data transmission: An idea whose time has come," IEEE Commun. Mag., vol.28, no.5, pp.5-14, May 1990.

[3] B. Sheng and X. You, "Joint estimation of Doppler spread and carrier frequency offset for OFDM systems," IEICE Trans. Fundamentals, vol.E88-A, no.11, pp.3134-3136, Nov. 2005.
[4] Y.H. You, S.J. Kang, D.K. Hong, J.Y. Lee, and J.W. Cho, "Estimation of OFDM integer frequency offset over rapidly time-varying channels," IEICE Trans. Commun., vol.E89-B, no.7, pp.2099-2101, July 2006.

[5] G. Mkrtchyan, K. Naito, K. Mori, and H. Kobayashi, "Doppler spread estimation method for OFDM signal using mean square of channel impulse response's time derivative," IEICE Trans. Commun., vol.E89-B, no.10, pp.2961-2966, Oct. 2006.

[6] S. Ye, R.S. Blum, and L.J. Cimini, Jr., "Adaptive modulation for variable-rate OFDM systems with imperfect channel information," Proc. IEEE VTC Spring 2002, vol.2, pp.767-771, May 2002.

[7] T. Usui, F. Ishizu, and K. Murakami, "A study on adaptive modulation technique in OFDM," IEICE Trans. Commun. (Japanese Edition), vol.J88-B, no.3, pp.634-642, March 2005.

[8] W.Y. Zou and Y. Wu, “COFDM: An overview," IEEE Trans. Broadcasting, vol.41, no.1, pp.1-8, March 1995.

[9] IEEE P802.16-REVd - Standard for Local and Metropolitan Area Networks - Part 16: Air Interface for Fixed Broadband Wireless Access Systems.

[10] Y. Zhao and S.G. Haggman, "Intercarrier interference selfcancellation scheme for OFDM mobile communication systems," IEEE Trans. Commun., vol.49, no.7, pp.1185-1191, July 2001.

[11] F. Sasamori, Y. Ishikawa, S. Handa, and S. Oshita, "A simple expression of BER performance in COFDM systems over fading channels," IEICE Trans. Fundamentals, vol.E92-A, no.1, pp.332-336, Jan. 2009.

[12] Y. Ma, Y. Huang, F. Liu, and N. Yi, "Blind channel estimation for repetition coded OFDM in block Rayleigh fading," Proc. IEEE GLOBECOM2003, vol.1, pp.30-34, Dec. 2003.

[13] Y.H. Kim, I. Song, H.G. Kim, T. Chang, and H.M. Kim, "Performance analysis of a coded OFDM system in time-varying multipath Rayleigh fading channels," IEEE Trans. Veh. Technol., vol.48, no.5, pp.1610-1615, Sept. 1999.

[14] J. Zheng, and S.L. Miller, "Performance analysis of coded OFDM systems over frequency-selective fading channels," Proc. IEEE GLOBECOM2003, vol.3, pp.1623-1627, Dec. 2003.

[15] S.M. Alamouti, "A simple transmit diversity technique for wireless communications," IEEE J. Sel. Areas Commun., vol.16, no.8, pp.1451-1458, Oct. 1998.

[16] D. Agrawal, V. Tarokh, A. Naguib, and N. Seshadri, "Space-time coded OFDM for high data-rate wireless communication over wideband channels," Proc. IEEE VTC'98, vol.3, pp.2232-2236, May 1998.

[17] K.F. Lee and D.B. Williams, "A space-frequency transmitter diversity technique for OFDM systems," Proc. IEEE GLOBECOM'00, vol.3, pp.1473-1477, Nov. 2000.

[18] P.A. Bello, "Characterization of randomly time-variant linear channels," IEEE Trans. Commun. Syst., vol.CS-11, no.4, pp.360-393, Dec. 1963.

[19] W.C. Jakes, Microwave Mobile Communications, IEEE Press, New York, 1974.

[20] Z. Jia, S. Handa, F. Sasamori, and S. Oshita, "Multiple-symbol differential detection for unitary space-time-frequency coding," IEICE Trans. Commun., vol.E93-B, no.1, pp.90-98, Jan. 2010.

[21] BRAN WG3 PHY Subgroup, "Criteria for comparison," ETSI/ BRAN document no.30701F, 1998.

[22] F. Sasamori, S. Handa, and S. Oshita, "A simple method of BER calculation in DPSK/OFDM systems over fading channels," IEICE Trans. Fundamentals, vol.E88-A, no.1, pp.366-373, Jan. 2005.

[23] J.G. Proakis, Digital Communications, McGraw-Hill, New York, 1983.

[24] Y. Karasawa, Radiowave propagation fundamentals for digital mobile communications, Corona Publishing, Tokyo, 2003.

[25] H. Futaki and T. Ohtsuki, "Performance of low-density paritycheck (LDPC) coded OFDM systems," Proc. IEEE ICC 2002, vol.3, pp.1696-1700, May 2002. 


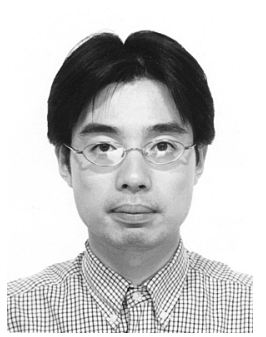

Fumihito Sasamori received the B.E., M.E. and Dr.Eng. Degrees from Waseda University, Tokyo in 1994, 1996 and 2000, respectively. Since 2000 he has been with the Department of Electrical and Electronic Engineering, Shinshu University, first as a Research Associate and since 2006 as an Associate Professor. His current research interests include digital mobile communication systems. He received the IEICE Young Engineer Award in 2000. He is a member of IEEE.

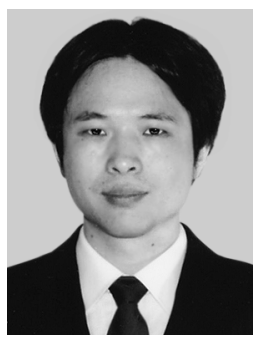

Ziyan Jia received the B.E. degree from Nanjing University of Posts and Telecommunications, China in 2004, and the M.E. and Dr.Eng. degrees from Shinshu University, Japan in 2007 and 2010, respectively. He has been with the center of researchers' training for promoting innovation, Shinshu University, since 2010 as a postdoctoral researcher. His current research interests include coding and modulation for mobile communications.

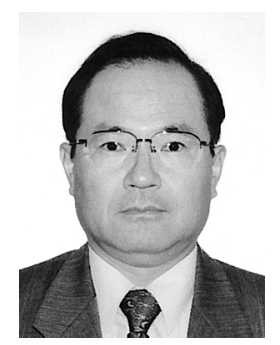

Shiro Handa received the B.E. and M.E. degrees from Shinshu University in 1978 and 1980 respectively, and the Dr.Eng. degree from Kobe University in 1988. From 1982 to 1988, he was a Research Associate at Kobe University. From 1988 to 1994, he was with Nagano National College of Technology. He has been with the Department of Electrical and Electronic Engineering, Shinshu University, since 1994 as an Associate Professor and since 2005 as a Professor. In 1996, he was at the University of California,

Davis, as a visiting researcher. His research interests include satellite and mobile communication systems, modulation and coding, and data compression. He is a member of IEEE and SITA.

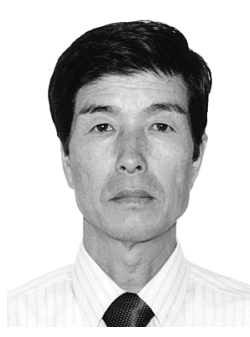

Shinjiro Oshita received the B.E. degree from Osaka Electro Communication University in 1967, the M.E. and Dr.Eng. degrees from Osaka University, Japan in 1970 and 1973, respectively. Since 1973, he has been with Shinshu University, Nagano, Japan, where he is currently a Professor Emeritus of Shinshu University. His research interests include intelligent $\mathrm{CAI}$ and communications theory. $\mathrm{He}$ is a member of IEEE and Japanese Society for Information and System in Education. 